\title{
Análise fitoquímica e avaliação da atividade fotoprotetora do extrato aquoso de
}

\section{Tamarindus indica L. (Tamarindo)}

Phytochemical analysis and evaluation of the photoprotective activity of the aqueous extract of Tamarindus indica L. (Tamarindo)

Analisis fitoquimico y evaluacion de la actividad fotoprotectora del extracto acuoso de Tamarindus

indica L. (Tamarindo)

Maria Auxiliadora da Silva Ramalho

ORCID: https://orcid.org/0000-0001-9523-6347

Universidade Federal de Campina Grande, Brasil

E-mail: mary.cylya06@gmail.com

Maria Francyherla Miguel da Silva Leite ORCID: https://orcid.org/0000-0002-7968-7751

Universidade Federal de Campina Grande, Brasil E-mail: francyherllaleiite@gmail.com

Mylena Medeiros Simões ORCID: https://orcid.org/0000-0002-0654-4733 Universidade Federal de Campina Grande, Brasil

E-mail: mylenamedeirossimoes@ gmail.com

Ozivaldo Ferreira de Souza

ORCID: https://orcid.org/0000-0002-4888-2116 Universidade Federal de Campina Grande, Brasil E-mail: ozivaldo321@gmail.com

Rayssa Ferreira de Lima ORCID: https://orcid.org/0000-0002-3047-8772 Universidade Federal de Campina Grande, Brasil E-mail: rayssafpb@gmail.com

Thayná Kelly Formiga de Medeiros ORCID: https://orcid.org/0000-0002-7540-1293 Universidade Federal de Campina Grande, Brasil E-mail: thaynak98@gmail.com

Raline Mendonça dos Anjos ORCID: https://orcid.org/0000-0003-0751-7523 Universidade Federal de Campina Grande, Brasil E-mail: raline.anjos@gmail.com

Luciano de Brito Júnior ORCID: https://orcid.org/0000-0002-1787-4316 Universidade Federal de Campina Grande, Brasil E-mail: lbritojunior@hotmail.com

Gymenna Maria Tenório Guênes ORCID: https://orcid.org/0000-0002-5447-0193 Universidade Federal de Campina Grande, Brasil E-mail: gymennat@yahoo.com.br 


\title{
Resumo
}

O presente estudo teve como objetivo realizar a análise fitoquímica e avaliar o fator de proteção solar (FPS) do extrato aquoso das cascas do fruto de Tamarindus indica L. (Tamarindo). Foi determinado o perfil fitoquímico através da metodologia utilizada por Matos (1997); o FPS in vitro através da metodologia de Mansur et al. (1986) através da realização de varreduras com o auxílio do espectrofotômetro (290 a 320nm) com intervalos de 5nm em concentrações de $(50,100,500$ e $1000 \mu \mathrm{g} / \mathrm{mL})$ do extrato aquoso do Tamarindus indica L. Os resultados obtidos evidenciaram que as concentrações de 50 e $500 \mu \mathrm{g} . \mathrm{mL}-1$ apresentaram o potencial fotoprotetor com FPS de 6,54 e 12,77, respectivamente. Com base nesses dados posso concluir que o extrato aquoso das cascas do fruto do Tamarindus indica L. é um promissor produto natural para uma possível utilização como protetor solar, tendo em vista que este possui como metabólitos secundários alcaloides e taninos, moléculas com bom potencial biológico.

Palavras-chave: Tamarindus indica L; Radiação UV; Fotoproteção.

\begin{abstract}
The present study aimed to perform the phytochemical analysis and evaluate the sun protection factor (SPF) of the aqueous extract of the fruit peels of Tamarindus indica L. (Tamarindo). The phytochemical profile was determined through the methodology used by Matos (1997); the FPS in vitro through the methodology of Mansur et al. (1986) by performing scans with the aid of a spectrophotometer (290 to 320nm) with $5 \mathrm{~nm}$ intervals in concentrations of (50, 100,500 and $1000 \mu \mathrm{g} / \mathrm{mL}$ ) of the aqueous extract of Tamarindus indica L. The results obtained showed that concentrations of 50 and $500 \mu \mathrm{g} . \mathrm{mL}-1$ showed the photoprotective potential with SPF of 6.54 and 12.77 , respectively. Based on these data, I can conclude that the aqueous extract from the peels of the Tamarindus indica L. fruit is a promising natural product for possible use as a sunscreen, considering that it has alkaloids and tannins as secondary metabolites, molecules with good biological potential.
\end{abstract}

Keywords: Tamarindus indica L; UV radiation; Photoprotection.

\section{Resumen}

El presente estudio tuvo como objetivo realizar el análisis fitoquímico y evaluar el factor de protección solar (FPS) del extracto acuoso de las cáscaras de fruta de Tamarindus indica L. (Tamarindo). El perfil fitoquímico se determinó mediante la metodología utilizada por Matos (1997); Metodología in vitro SPF de Mansour et al. (1986) realizando exploraciones con la ayuda de un espectrofotómetro $(290$ a $320 \mathrm{~nm}$ ) con intervalos de $5 \mathrm{~nm}$ en concentraciones de $(50,100,500$ y $1000 \mu \mathrm{g} / \mathrm{ml})$ del extracto acuoso de Tamarindus indica L. Los resultados obtenidos mostraron que concentraciones de 50 y $500 \mu \mathrm{g} \cdot \mathrm{mL}-1$ mostraron el potencial fotoprotector con FPS de 6.54 y 12.77 , respectivamente. Con base en estos datos, puedo concluir que el extracto acuoso de las cáscaras de la fruta Tamarindus indica L. es un producto natural prometedor para su posible uso como protector solar, teniendo en cuenta que tiene alcaloides y taninos como metabolitos secundarios, moléculas con buen potencial biológico.

Palabras clave: Tamarindus indica L; Radiación UV; Fotoprotección.

\section{Introdução}

A Radiação Ultravioleta (R-UV) possibilita a síntese de vitamina D na pele humana, no entanto, a exposição quando de maneira excessiva pode causar danos à saúde (Oliveira, 2013). De acordo com Okuno e Vilela (2005), pode causar uma série de danos à saúde humana tais como o envelhecimento precoce, a deleção do sistema imunológico, catarata e o câncer de pele, dentre outros.

Segundo o Centro de Oncologia do Hospital Alemão Oswaldo Cruz (2016), a exposição à radiação solar em excesso e a ausência de proteção são os principais fatores de risco para o desenvolvimento do câncer de pele, provocando alterações celulares, pessoas de pele clara, com pintas e manchas, idosas e as que possuem histórico de câncer de pele na família estão mais propícios a desenvolver a doença, bem como, aquelas que também muito se expôs ao sol ao longo da vida. 
Sendo assim, fazem-se necessárias algumas precauções, como as mencionadas pelo INCA (2017): evitar exposição prolongada ao sol entre 10h e 16h (considerados os mais intensos), caso a exposição seja inevitável, deve-se procurar lugares com sombra (proteções físicas), usar proteção adequada (como roupas, bonés e óculos escuros com proteção UV) além do uso de filtro (protetor) solar. Logo, esses cuidados são indispensáveis desde a infância, pois se acredita que os danos ocasionados pelo abuso de exposição solar são cumulativos (INCA, 2019).

No entanto, vale salientar que qualquer pessoa pode desenvolver o câncer de pele, desse modo, o uso de fotoprotetores contra a radiação ultravioleta além de ser uma proteção viável e vantajosa para os diversos tipos de pele, está disponível em soluções cosméticas para uso tópico, implicando filtros solares químicos e/ou físicos (Billhimer,1998, ANVISA,2002). Uma das opções é o desenvolvimento de fotoprotetores a base de produtos naturais de plantas vegetais.

Uma família muito rica na flora brasileira é a Fabaceae, constitui uma das maiores famílias de angiospermas, com 727 gêneros e cerca de 19.325 espécies, distribuídas em três subfamílias: Faboideae, Mimosoideae e Caesalpinioideae (Lewis et al., 2005). A família apresenta uma distribuição ampla ao redor do globo, está presente tanto nas florestas tropicais quanto desertos, planícies e regiões alpinas (Doyle \& Luckow 2003). Apresentando representantes dos mais diversos tipos de hábito: árvores, arbustos, lianas e até plantas aquáticas.

Nessa família, encontra-se a espécie Tamarindus indica L., as cascas do tamarindo possui característica adstringente e tônica são de coloração marrom, frágeis, quebradiças e lenhosas. Vários estudos têm revelado que as cascas e as sementes do fruto exibem um teor relevante de fitoquímicos bioativos, como, flavonoides, antocianinas, carotenoides, compostos fenólicos (Jardini \& Macinni-Filho, 2007; Melo \& Andrade, 2010; Almeida et al., 2016).

A espécie Tamarindus indica L., vulgarmente conhecida como tamarindo, é uma árvore com origem cultivada, presente na Caatinga (Domínio Fitogeográfico), com distribuição no Norte, Centro-Oeste, Sudeste, Sul e grande parte do Nordeste (Alagoas, Bahia, Ceará, Maranhão, Paraíba, Pernambuco, Rio Grande do Norte e Sergipe) (Flora do Brasil, 2020).

Os resíduos de frutas (cascas e sementes) podem conter um número maior de compostos bioativos do que as suas respectivas partes comestíveis (Soares et al., 2008; Alves et al., 2013; Goldmeyer et al., 2014). Os quais são amplamente reconhecidos pelas suas propriedades promotoras de saúde e aplicações tecnológicas, como antioxidantes e antimicrobianos, representando, portanto, potenciais fontes naturais dessas substâncias (Jorge \& Malacrida, 2008).

Tendo em vista a importância da espécie e de sua família, além da ausência de estudos quanto a atividade fotoprotetora do Tamarindo, o objetivo deste artigo foi realizar a análise fitoquímica e avaliar a atividade fotoprotetora do extrato aquoso das cascas do fruto de Tamarindus indica L. (tamarindo).

\section{Metodologia}

\section{Extrato vegetal}

O material para a realização do estudo in vitro foram às cascas do fruto da espécie Tamarindus indica L., coletado e identificado. Ademais, uma exsicata com numeração 7386 foi depositada ao acervo do Herbário CSTR (Centro de Saúde e Tecnologia Rural) Rita Baltazar de Lima da Universidade Federal de Campina Grande, campus Patos-PB. Para a produção do extrato aquoso das cascas do fruto do tamarindo, utilizou-se a metodologia descrita por Ferris \& Zheng (1999), com modificações.

\section{Perfil fitoquímico de Tamarindus indica $\mathbf{L}$.}

Para a realização da análise do perfil fitoquímico do extrato aquoso das cascas do fruto de Tamarindus indica L. utilizou-se a metodologia de Matos (1997), com modificações. 


\section{Avaliação do Fator de Proteção Solar do extrato aquoso de Tamarindus indica L.}

A espectrofotometria de absorção do extrato aquoso de Tamarindus indica L. aconteceu no espectro da radiação ultravioleta como proposto por Mansur et al., (1986), assim realizou-se varreduras de 290 a 320nm (em intervalos de $5 \mathrm{~nm}$ ) com duração de 5 minutos, sendo que ao término desse tempo foi efetuado as mensurações das absorbâncias. Para a leitura utilizou-se o espectrofotômetro digital (Biospectroß) com cubeta de quartzo de 1cm. Após a mensuração das absorbâncias, os dados foram submetidos à equação de Mansur e colaboradores (1986) para aferir o FPS in vitro. Esse método coloca em lista o efeito eritematogênico e a intensidade da radiação (EE X I) que foram medidos por Sayre et al., (1979). Esses são demostrados no Quadro 1, logo abaixo:

Quadro 1 - Relação efeito eritemogênico (EE) versus intensidade da radiação (I) conforme o comprimento de onda ( $\chi$ ).

\begin{tabular}{|c|c|}
\hline$\lambda / \mathbf{n m}$ & EE x I \\
\hline 290 & 0,0150 \\
\hline 295 & 0,0817 \\
\hline 300 & 0,2874 \\
\hline 305 & 0,3278 \\
\hline 310 & 0,1864 \\
\hline 315 & 0,0839 \\
\hline 320 & 0,0180 \\
\hline
\end{tabular}

Fonte: Sayre et al. (1979).

Sendo que a fórmula de Mansur et al. (1986) é também composta pela leitura espectrofotométrica da absorbância da solução e fator de correção (=10). Essa equação pode ser observada, a seguir:

FPS espectrofotométrico $=$ FC. $\Sigma \operatorname{EE}(\lambda) . \mathrm{I}(\lambda)$.

$\operatorname{Abs}(\lambda)$

Na qual: FPS = fator de proteção solar; FC = fator de correção, calculado de acordo com dois filtros solares de FPS conhecidos e testados em seres humanos de tal forma que um creme contendo $8 \%$ de homossalato resultasse no FPS 4; $\operatorname{EE}(\lambda)=$ efeito eritemogênico da radiação de comprimento de onda; $I(\lambda)=$ a intensidade da luz solar no comprimento de onda e Abs $(\lambda)$ = a absorbância da formulação no comprimento de onda.

\section{Resultados e Discussão}

Cresce cada vez mais o interesse pela busca de recursos naturais no ramo terapêutico. O uso de plantas com fim terapêutico possui ampla aceitação popular e apoio da Organização Mundial de Saúde (OMS), seu uso tem sido crescente, motivando pesquisas científicas (Souza et al., 2018).

Os metabólitos secundários de origem vegetal são responsáveis pela promoção da saúde e prevenção de uma série de doenças humanas. Por esse motivo, esses compostos podem contribuir para estabelecer a eficácia e os mecanismos desencadeadores de suas diversas ações. (Saxena et al., 2013).

Segundo Rosa et al., (2016), pesquisas fitoquímicas são de extrema utilidade, pois indicam a existência dos vários compostos que podem provocar diversos efeitos nos organismos expostos aos mesmos. 
Dessa forma, a análise fitoquímica do extrato aquoso das cascas de Tamarindus indica L., exposta na Tabela 1, indica a presença dos metabólitos secundários alcaloides e taninos.

Tabela 1. Análise fitoquímica do extrato aquoso das cascas do fruto de Tamarindus indica L.

\begin{tabular}{ll}
\hline Alcaloides & + \\
Esteroides e triterpenoides & - \\
Flavonoides & - \\
Saponina espumídica & - \\
Taninos & + \\
\hline
\end{tabular}

Legenda: (+) presença; (-) ausência. Fonte: Autores.

Os alcaloides são substâncias nitrogenadas, geralmente farmacologicamente ativas, e encontradas em plantas, predominantemente nas angiospermas. (Oliveira, 2008). É possível que esses metabolitos apresentem entre suas funções a defesa contra os raios ultravioleta, visto que, em sua maior parte, serem compostos com núcleos aromáticos que possui alto poder de absorção desses raios (Henriques et al., 2000).

Segundo El-Siddig et al., (2006) verificaram que a casca de tamarindo pode conter até $70 \%$ de taninos. Isto é importante tendo em vista que a presença de taninos manifesta potencial para absorver a radiação UV (Santana et al., 2001). A ausência de flavonoides no teste fitoquímico deste estudo pode ser justificado pelo uso do solvente extrator água, que tem baixa capacidade de arrastar esses metabólitos (Santos et al., 2018).

Quanto ao fator de proteção solar (FPS), vários estudos demonstram que os produtos naturais são a principal razão da diversidade química no decorrer do processo de descoberta no ramo farmacêutico. (Firn \& Jones, 2003; Mishra \& Tiwari, 2011). Os extratos são considerados significativos produtos, e são, normalmente, usados com fins farmacológicos, industriais e nutricionais. (Souza et al., 2013).

Observa-se a análise da atividade fotoprotetora do extrato aquoso das cascas do fruto de Tamarindus indica $\mathrm{L}$. realizada no espectro da radiação UVB, na Tabela 2.

Tabela 2. FPS do extrato aquoso das cascas do fruto de Tamarindus indica $\mathrm{L}$.

\begin{tabular}{ccc}
\hline Concentrações $(\boldsymbol{\mu g} . \mathbf{m L}-1)$ & $\mathbf{5 0} \boldsymbol{\mu g} / \mathbf{m L}$ & $\mathbf{5 0 0 \mu g} / \mathbf{m L}$ \\
\hline FPS & 6,19 & 12,77 \\
\hline
\end{tabular}

Fonte: Dados da pesquisa (2020).

Na avaliação espectrofotométrica do extrato aquoso na faixa de radiação UVB (290 a 320 nm), os resultados obtidos demonstraram valores relevantes para o estudo, uma vez que o extrato apresentou absorbâncias significativas nas concentrações de $50 \mu \mathrm{g} . \mathrm{mL}-1$ e $500 \mu \mathrm{g} . \mathrm{mL}-1$, apresentando potencial fotoprotetor com FPS de 6,54 e 12,77; respectivamente.

Tendo em vista a resolução - RDC No 30, de $1^{\circ}$ de junho de 2012, da Agência Nacional de Vigilância Sanitária (BRASIL, 2012), que aprova o regulamento técnico MERCOSUL sobre protetores solares em cosméticos e dá outras deliberações, o fator mínimo de proteção solar estabelecido é 6 (seis). Logo, a partir desses resultados propõe-se que o extrato aquoso das cascas do fruto de Tamarindus indica L. nas concentrações de 50 e $500 \mu \mathrm{g} . \mathrm{mL}-1$ poderiam ser utilizadas como protetores solares em fitocosméticos. 
Conforme o estudo de Simões et al., (2019a). O extrato etanólico de Gossypium hirsutum L. (algodão), nas concentrações de $500 \mu \mathrm{g} . \mathrm{mL}-1$ e $1000 \mu \mathrm{g} . \mathrm{mL}-1$, apresentou relevante potencial fotoprotetor com FPS de 16,17 e 25 , nessa ordem.

De acordo com a pesquisa de Simões et al., (2019b) O extrato etanólico das folhas de Cnidoscolus quercifolius Pohl, apresentou potencial fotoprotetor 6,48 e 14,30 nas concentrações de $500 \mu \mathrm{g} . \mathrm{mL}-1$ e $1000 \mu \mathrm{g} . \mathrm{mL}-1$, por essa ordem.

\section{Considerações Finais}

Com base nos resultados obtidos nesse trabalho, é possível concluir que o extrato aquoso das cascas do fruto do Tamarindus indica L. apresenta significante potencial fotoprotetor. Considerando-se um promissor produto natural para uma possível utilização como protetor solar. Sendo assim, uma das alternativas para o controle e prevenção de possíveis danos provocados pela luz solar. No entanto, faz-se viável e necessário intensificar os estudos sobre o assunto, como a realização de estudos in vivo para o desenvolvimento de um fitocosmético.

\section{Referências}

Agência Nacional de Vigilância Sanitária (2002). Regulamento técnico sobre protetores solares em cosméticos (RDC № 237 de 22 de agosto de 2002 ).

Almeida, M. L. B., Freitas, W. E. S., Morais, P. L. D., Sarmento, J. D. A., \& Alves, R. E. (2016). Bioactive compounds and antioxidant potential fruit of Ximenia americana L. Food Chemistry, 191, 1078-1082.

Alves, A. M., Alves, M. S. O., Fernandes, T. O., Naves, R. V., \& Naves, M. M. V. (2013). Caracterização física e química, fenólicos totais e atividade antioxidante da polpa e resíduo de gabiroba. Revista Brasileira de Fruticultura. 35(3).

Billhimer, W. L. (1998). Avaliação dos filtros solares em seres humanos: proteção contra a queimadura solar. Cosmetics \& Toiletries, 1, 41-8.

Brasil. (2012). Diário Oficial [da] República Federativa do Brasil. Aprova o regulamento técnico "Mercosul sobre Protetores Solares em Cosméticos e dá outras providências (Resolução RDC $\mathrm{n}^{\mathrm{o}}$ 30, de 1 de junho de 2012). Obtido a partir de http://bvsms.saude.gov.br/ bvs/saudelegis/anvisa/2012/rdc0030_01_06_2012.html.

Doyle, J. J., \& Luckow, M. (2003). The rest of the iceberg- Legume diversity and evolution in a phylogenetic context. Plant Physiology, 131, 900-910.

El-siddig, K., Gunasena, H. P. M., Prasa, B. A., Pushpakumara, D. K. N. G., Ramana, K. V. R., Vijayanand. P., \& Williams, J. T. (2006). Tamarind Tamarindus indica L. Fruits for the future 1. Southampton Centre for Underutilized Crops, Southampton, UK. p.188.

Fabaceae in Flora do Brasil (2020) em construção. Jardim Botânico do Rio de Janeiro. http://reflora.jbrj.gov.br/reflora/floradobrasil/FB23200. Acesso em: 24 março 2020.

Ferris, H., \& Zheng, L. (1999). Plant sources of chinese herbal remedies: effects on Pratylenchus vulnus and Meloidogyne javanica. Journal of nematology, Lawrence, p.241-263.

Firn, R. D., \& Jones, C. G. (2003). Natural products-a simplemodel to explain chemical diversity. Natural productreports, 20 (4), $382-391$.

Goldmeyer, B., Penna, N. G., Melo, A., \& Rosa, C. S. (2014). Características físico-químicas e propriedades funcionais tecnológicas do bagaço de mirtilo fermentado e suas farinhas. Revista Brasileira de Fruticultura, 34(4).

Henriques, A. T., Kerber, V. A., \& Moreno, P. R. H. (2000). Alcalóides: generalidades e aspetos básicos. Farmacognosia: da planta ao medicamento, (2), 641-642.

INCA. (2017). Câncer: a informação pode salvar vidas. Comunicação Social, p.2.

INCA. (2019). Como se proteger do câncer de pele. https://www.inca.gov.br/causase-prevencao/prevencao-e-fatores-de risco/exposicaosolar/como-seproteger-do-cancer-de-pele. Acesso em: 24 mar, 2020.

Jardini, F. A., \& Mancini-Filho, J. (2007). Avaliação da atividade antioxidante em diferentes extratos da polpa e sementes da romã (Punica granatum, L.). Revista Brasileira de Ciências Farmacêuticas, 43(1).

Jorge, N., \& Malacrida, C. R. (2008) Extratos de sementes de mamão (Carica papaya L.) como fonte de antioxidantes naturais. Alimentos e Nutrição, 19, 337340 .

Lewis, G., Schrire, B., Mackinder, B. \& Lock, M. (2005). Le-gumes of the world. Kew: Royal Botanic Gardens, p. 577.

Luzia, D. M. M., \& Jorge, N. (2011). Antioxidant activity, fatty acid profile and tocopherols of Tamarindus indica L. seeds. Ciência e Tecnologia de Alimento, 31(2), 497-501. 
Research, Society and Development, v. 10, n. 9, e25810917985, 2021

(CC BY 4.0) | ISSN 2525-3409 | DOI: http://dx.doi.org/10.33448/rsd-v10i9.17985

Mansur, J. D. S., Brender, M. N. R., \& Mansur, M. C. D. A. (1986). Determinação do fator de proteção solar por espectrofotometria. Anais brasileiros de dermatologia. 61(3), 121-124.

Matos, F. J. A. (1997). Introdução à fitoquímica experimental. UFC. (2)

Melo, E. A., \& Andrade, R. A. M. S. (2010). Compostos bioativos e potencial antioxidante de frutos de umbuzeiro. Revista Alimentos e Nutrição, 21(3), 453457.

Mishrs, B. B., \& Tiwari, V. K. (2011). Natural products: anevolving role in future drugdiscovery. European journal of medicinal chemistry, 46(10), 47694807

Okuno, E. \& Vilela, C. A. P. (2005). Radiação Ultravioleta: Características e Efeitos. Livraria da Física.

Oliveira, M. M. F. (2013). Radiação ultravioleta/ índice ultravioleta e câncer de pele no Brasil: condições ambientais e vulnerabilidades sociais. Revista Brasileira de Climatologia, 13.

Oliveira, V. B. (2008). Alcaloides indólicos de Aspidosperma spruceanum (Apocynaceae). Tese (Doutorado em Produção Vegetal). Universidade Estadual do Norte Fluminense Darcy Ribeiro - UEFN -Campos Dos Goytacazes, p.104.

Oswaldo Cruz. (2016). Excesso de exposição à radiação solar é o principal causador da doença. https://centrodeoncologia.org.br/noticias-cancer/excesso-deexposicao-radiacao-solar-e-o-principal-causador-da-doenca.

Rosa, R. C. A. et al. (2016). Triagem fitoquímica dos extratos aquosos de Bauhinia candicans, Foeniculum vulgare, Mentha pulegium e Morus nigra. Conexão Ciência (Online). 5(1), 44-51.

Santana, J. L. et al. (2001). Avaliação da atividade antimicrobiana, fotoprotetora, antielastase e antioxidante de polifenois de origem natural, aplicada com formulações cosméticas. XV Congresso Latino-americano e Ibérico de Químicos Cosméticos. Buenos Aires, Argentina, p. 332-343.

Santos, T. R. J. et al. (2018). Avaliação de diferentes solventes na extração de compostos bioativos em resíduos de Tamarindo (Tamarindus indica). Simpósio Internacional de Inovação Tecnológica, 8(1), 124-131.

Saxena, M., Saxena, J., Nema, R., Singh, D., \& Gupta, A. (2013). Phytochemistry of medicinal plants. Journal of Pharmacognosy and Phytochemistry, 1(6), 168-182.

Sayre, R. M. et al. (1979). A comparison of in vivo and in vitro testing of sunscreening formulas. Photochemistry and Photobiology, 29(3), 559-566.

Simão, K. L. A. et al. (2019a). Avaliação do potencial fotoprotetor do extrato etanólico das folhas de cnidoscolus quercifolius pohl. Revista International Journal of Development Research. 09, (09), 29883-29886.

Simão, K. L. A. et al. (2019b). Estudo fotoprotetor do extrato etanólico das partes aéreas de Gossypium hirsutum L. (algodão). Revista Brasileira de Educação e Saúde. 9(4), 45-49.

Soares, M., Welter, L., Kuskoski, E. M., Gonzaga, L., \& Fett, R. (2008). Compostos fenólicos e atividade antioxidante da casca de uvas Niágara e Isabel. Revista Brasileira de Fruticultura, 30(1), 59-64.

Souza, D. P. M. D. S., Galdino, O. R., \& Lopes, D. S. (2018). O uso fitoterápico no tratamento da doença periodontal.

Souza, F. P. de, Campos, G. R., \& Packer, J. F. (2013). Determinação da atividade fotoprotetora e antioxidante em emulsões contendo extrato de Malpighia glabra L. - Acerola. Revista de Ciências Farmacêuticas básica e aplicada, 34(1), 69-77. 